PIOTR ŁUKOWSKI

\title{
TWO PROCEDURES EXPANDING A LINGUISTIC COMPETENCE
}

\section{Introduction}

On the ground of psychology there are many various theories explaining what a notion is. All these approaches can be grouped in three classes:

(A) classical theories which present a notion as a compilation of features in common for many particular objects.

(B) probabilistic theories in which notions are derived from the higher structure - an individual theory of the world. Notions are elements of the structure.

(C) theories in which a notion is strictly connected with its references and with other notions.

Theories of the first class are a little bit naïve today, and theories of the second class fail because of the error of circularity - in order to explain what a notion is, we need to explain what a structure is, but in order to explain what a structure is, we need to know what a notion is (Maruszewski, 2001: 297-299). Theses of theories of the third class are obviously compatible with simple everyday observations and with the logical knowledge about the properties of natural language expressions. ${ }^{1}$ Thus, the approach presented in the paper coincides with the main ideas of the theories of this last class.

${ }^{1}$ In his book Concepts, Kinds and Cognitive Development Keil considers the process of concepts creation in child's mind. Both the dialogues to be presented below, well known from our life, as well as the conclusions of my paper coincide with basic thesis by Keil. For example, in his theory Keil employs known in psychology notion "zone of proximal development" (created by famous psychologist Lev Vygotsky) as a name for all those people which help us to understand and to correctly use new (for us) words. Also, the notion of similarity, used by us in the paper, is more precisely analyzed by another famous psychologists Inhelder and Piaget. They consider a development of a child's mind 


\section{Dialogue 1}

During a stroll with his father a boy asks: - What is it, daddy?

- A building, honey - the father answers.

- Oh...

- And this is another building, isn't it? - after a while the child adds pointing at next object.

- No, it isn't. This is a shed.

- Why this is not a building? - the boy asks.

- Because it is much smaller than a building, it is constructed from ordinary boards, it is not for living, just for garden's tools...

- Uh-huh...

\section{Dialogue 2}

During a stroll with his father a child asks: - What color is this leaf?

- It is brown - the father answers.

- It's impossible! Your jacket is brown and the leaf's color is different.

- My jacket is brown and the leaf is also brown. Both colors are a little bit different but both are brown - the father states.

- And what about that car's color? - the boy asks.

- I'm afraid the car is not brown - the father answers - that car's color has a delicate red shade.

Every story is well known and typical for strolling with a small child. Both present a similar process of expanding the linguistic competence of a child. In both cases the child is taught by his father how to use words correctly. In the first story the child recognizes a correct use of the word "building" and the correct use of the word "shed". In the second story the child is taught by his father how to correctly use the word "brown". In spite of similarities both processes fundamentally differ. In the first scene, two objects named by the child (with the father's help) differ in some clear, easy to notice way, and that is why the difference can be defined. Obviously, the definition can be partial only. As far as the terms are not mathematical defining cannot be complete - it can be complete only for the terms of a mathematical, strict sense. In the second scene the differences between colors of objects are subtle and difficult to describe. In this case, a correct use of the name depends on the accurate recognition of resemblance to the model object.

as a process in which concept creation firstly bases on "accidental similarities" of objects, but later on "essential similarities”, see (Keil, 1989: 5-7, 9), also (Piaget, Inhelder, 1974: 37). 
Both simple and standard stories illustrate a process of learning of the correct use of new words. Thanks to them, in a schematic way, the most basic truths are shown about the process. The careful examination of both situations should deliver some important hints about the modeling of this process for automata. Thus, first of all let us notice that the entire process depends on the permanent teaching of the child by the parent. In other words, it is necessary to continuously verify the process. The process is controlled from outside and depends on the regular provision of new information about the correct use of words. Sometimes, a child is informed about a name of an object new for them, in other times an example is provided, i.e. an object which can be used by the child as a model object, an object which is a case of the correct use of the given word. It means that there is another important element of the process: an ostensive definition. Since the process in question consists in the permanent confrontation of words with non-linguistics objects, an ostensive defining must be a constant element of the process. During a conversation, a new word appears together with a gesture of pointing to an object which is an example either of the correct or of the wrong use of the word. Usually, in the ostensive defining the same person utters the word and shows an object. In our stories this kind of defining splits into two persons: the first one utters the word and the second points to an object. Sometimes the first person is a child, and the second a father, sometimes it is the other way round. Undoubtedly, in all those cases there is still ostensive defining.

Another constant element of the procedure that extends the linguistic competence is a partial defining of the meaning of new words. This fact is evident in the case of the first dialogue. However, the second situation is not free from the partial defining - here, the partial defining is of the ostensive type. In the first dialogue, the meaning (the content) of the word "shed" is distinguished from the meaning (the content) of "building" by using only few arbitrary features. Indeed, after the initial exchange at the beginning of the dialogue, a child believes that he knows how to correctly use the word "building". Then, he uses the word in a wrong way, calling "a building" an object that is not a building. The father corrects the boy calling a new object "shed" and giving some spontaneously selected features typical for sheds but not for buildings. Since this set of features is arbitrary it could be replaced by some other set. Of course, none of such sets can be used for a complete definition of the 
shed, because such a complete content (i.e. set of features) does not exist for words without mathematical sense. Obviously, such a step is repeated many times during a lifetime of each of us. We use some word already known to us in all cases similar to the model (for us) case, as long as somebody would correct us. Similarly, the child from the first dialogue possesses in his memory a model of a building being a picture of some real object, which was correctly named as "building". Then, the child tries to compare every newly encountered object to the picture of the building from his memory. If a similarity of both objects is in some sense sufficient, the child will call the new object, "a building". If not, the child will try to find an appropriate name for the new object. Probably, this will be done with the help of somebody: the father, the mother, some colleague, etc. In the first dialogue, the father gives his child a model example for the correct use of the word "shed". From now on, the boy possesses at least two model pictures: one representing "typical" building and another representing also "typical" shed. Moreover, a difference between a "typical" building and a "typical" shed is defined selectively, i.e. partially. During one's life, model examples evaluate (are changed many times). They are replaced by better, "more typical" objects. This permanent and endless improvement of the model cases is the result of the permanent and endless expansion of word's meaning understanding. Moreover, usually one model case for a given word is not enough and so the correct use of this word is recognized by more than one exemplary object. Thus, it should be supposed that after some time the child from the above stories (as well as everybody) possesses more than one example for the "typical" building and also more examples for the "typical" shed - after all, there are plenty of various buildings and many diverse sheds.

A partial defining appears also in the second dialogue. The child tries to know which color can be called "brown". As a result, the child possesses in his memory pictures of few brown objects: the father's jacket, the leave. He also knows that the car is not brown. It is an evident case of partial defining of the color. It is impossible for the child to know all shades of brown color, so all those cases are "partially" represented in the child's memory by those three examples: two positive and one negative.

In order to use the model examples for word's understanding and to use them correctly the child needs to competently recognize which features should be compared and which should be ignored - some features are essential, others

\section{O) COGNITIVE SCIENCE}


are inessential from the point of view of the word's meaning. In the case of the first dialogue, the child's successful use of the words "building" and "shed" depends on their ability to recognize which features are essential, and which are not, to distinguish buildings from sheds. Although the boy goes beyond the ostensive defining at this stage of the process, he employs all the results that have been engraved into his memory by the former ostensive defining.

Let us emphasize that the procedure that expands a linguistic competence by mathematical notions is quite different. Since those notions are precise and sharp, the parent or teacher has to present to the child their complete definitions. An incomplete definition would define some other notion, for example, a partial definition of the square "it is a two-dimensional figure with all four angles right and with opposite sides parallel” would define the rectangle.

\section{Generality - one face of the tolerance of expression}

A definition of a tolerant expression uses a class of functions of similarity thanks to which it is possible to recognize if a given new still unnamed object can be named by this expression i.e. if it is sufficiently similar to some of the images of objects being model examples of the correct use of this expression. Every function establishes the degree of similarity to at least one from all model examples from our memory. If there is, in our memory, some model example for the same name to which a new object is similar (it means that there is a sufficient degree of similarity), then this new object can be called by this name. Sometimes, for some names it is quite difficult to find only one model example. To illustrate, this is the case with the name "temple". Neither a concrete church nor a concrete synagogue nor a concrete mosque, nor a Buddhist temple, nor ... can be the model example for "temple". Pointing out to some concrete church as the model example of temple would suggest that neither a synagogue and nor a mosque is a temple. That is why in the case of some names we have more than one model example. It can be even assumed that we have more than one model example for most names we use. In the case of the name "temple" it is much easier to possess several model examples representing "typical" church, "typical" synagogue, "typical" mosque, "typical" Buddhist temple, and so on. The problem discussed here is typical for general names. A name is general if it has more than one reference. In other words the set of designates (a scope) of the general name has more than one 
element. Obviously, a set with more than one element can be divided into nonempty subsets. For every general name there are various principles of division of its scope. These principles depend on our interest. We can choose some interesting for us feature and divide the scope on elements possessing the feature or not. Thus, for the name "child" one can use the feature "born in Europe". Then, the set of all children will be divided into two subsets: all children born in Europe, and all other children. If the feature is gradable, we can divide the scope into subsets associated with each degree of the feature. For the same name "child" one can use gradable feature "born in the year $n$ ". In this case, the set of all children will be divided on subsets: children born in 2014, children born in 2013 ... It is easy to notice that every subset of the scope has its own name subordinate to the initial name. In general, the scope $a$ of the general name $A$ can be divided into subsets $a_{1}, \ldots, a_{n}$, associated with names $A_{1}, \ldots, A_{n}$, being subordinate to $A$. Thus, the name $A$ is superior to every name $A_{i}$, for $i=1, \ldots, n$. In Aristotelian logic, there is a tradition to call $a$ - the scope of the name $A$ - a genus, and its every subset $a_{i}$, for $i=1, \ldots, n, a$ species. If such division of the genus is possible it means than the name of the genus is general. It is the second understanding of the generality of the name. Obviously, both understandings are equivalent. Indeed, if the name has more than one designate it means that its scope can be divided into non-empty subsets, and conversely, if the scope of the name can be divided into non-empty subsets it means that it is a set with more than one element.

For every species (genus), there is a set of features or qualities such that every member of this species (genus) and no other object possesses all of these features or qualities. Every such feature/quality is called a feature/quality characteristic for the species (genus). The use of a general name is vulnerable to the error of generality (Łukowski, 2012: 92-93). This error has two variants. In the first one, a user of a general name $A$ attributes some feature or quality characteristic for one species $a_{i}$ to members of some other species $a_{j}$ (with $j \neq i$ ), because both species belong to the same genus $a$. In another version, a user attributes some feature or quality characteristic for one species $a_{i}$ to the entire genus $a$, because species $a_{i}$ belongs to $a$. For example, if somebody observed that his/her daughter likes to play with dolls, he/she believes that the boy in the neighborhood also likes to play with dolls (the first version of the error) or that all children like to play with dolls (the second version of the error). It seems that it is easier to avoid the error of generality in the situation when one model example for correct use of the name is replaced by several different model examples. Of course, such an 
approach does not guarantee avoiding this error - usually, the set of examples is not sufficient. On the other hand, even in the case of "temple" it is possible to partially define a temple as a building in which people gather to pray. Such species-free definition of the temple can be used in parallel with the definition using various species represented by the set of model examples. Obviously, both ways of defining are partial.

One of the most important features of a majority of expressions of the natural language is simple: every such expression must possess more than one reference - object, situation, etc. In other words, it must be useful in more than one case. This multiusage should be limited in some reasonable way - an expression which would fit all objects, situations, properties, and so on, would be useless. If the expression $A$ can be correctly used in the cases $P_{1}, \ldots, P_{n}$, then it should be correctly used also in all cases sufficiently similar to at least one of $P_{1}, \ldots, P_{n}$ (Eukowski, 2011: 133-134). Of course, the sufficient similarity is due to the meaning of the expression $A$. This is a definition of the tolerance of the expression $A$. This definition is strictly connected with the problem of generality. All cases $P_{1}, \ldots, P_{n}$ can be and should be understood as the model examples. On the other hand, every nonmathematical general name must be tolerant. Cases $P_{1}, \ldots, P_{n}$ mentioned in the definition can represent various species but do not have to - all of them can be model examples of one species only. But the most natural situation is one in which every $P_{i}$, for $i=1, \ldots, n$, represents a distinct species. In the case of the name $A=$ "temple", $P_{1}$ can be a concrete church, $P_{2}$ some concrete synagogue, $P_{3}$ a concrete mosque, and so on.

Obviously, every species of the genus can also be divided into some subspecies. Then, the divided species becomes a new genus and its subspecies, new species. Thus, for example, the scope of the general name "church" cab be divided into subsets composed of, respectively catholic churches, evangelical churches, orthodox churches... Obviously, in the next step, one can divide any scope of the new species, e.g. the set of catholic churches into subsets: catholic churches built in France, catholic churches built in Italy ... The division of the scope of the general name is impossible when the scope is a one-element set, e.g. the scope of the name "church located on the Wawel Hill". Usually, such a one-element set is represented by a proper name - in this case by "Wawel Cathedral".

In the definition of tolerant expression, there is another important element: similarity besides model objects/cases. This notion has two essential components: sufficiency of similarity and similarity due to the meaning of the (tolerant) 
expression. Both issues can be more easily explained on the colored objects. For this purpose let us imagine three paper leaves: $P_{1}$ - big white leaf overprinted by black letters on one side; $P_{2}$ - red leaf of the same size and shape as $P_{1}$ overprinted in the same way as $P_{1}$; and $P_{3}$ - small crumpled pale red leaf unwritten on both sides. It is obvious that every two leaves from the set $\left\{P_{1}, P_{2}, P_{3}\right\}$ are similar from some reasonable point of view. Moreover, every similarity is even sufficient in some sense but it does not mean that every two leaves are similar in general. Let us consider the name $A=$ "red leaf of paper". Surely, the second object can be called $A$, and so it can be also a model example for correct use of the name $A$. In order to know if $P_{1}$ or $P_{3}$ can be called $A$, we need to recognize if they are sufficiently similar to $P_{2}$, of course, due to the meaning of the general name $A$. Leaves $P_{1}$ and $P_{2}$ are similar - they are identical in size, both are overprinted in the same way by the same text or pattern. But $P_{1}$ is white while $P_{2}$ is red. Thus, if the similarity would be settled due to the color, they are not similar at all, and it does not matter if this non-similarity is sufficient or not. It means that, if $P_{2}$ is correctly called $A$, then $P_{3}$ cannot be called $A$ - since $P_{1}$ and $P_{2}$ are not sufficiently similar due to the red color, only one of these cases can be called red. However, the second comparison is not as easy as the first one. Obviously $P_{1}$ is red, but what about $P_{3}$ which is pale red? In this situation everything depends on our opinion based on our experience, knowledge, intuition, etc. If the pale-red color is in our opinion closer to red than to non-red, we will recognize that $P_{1}$ and $P_{3}$ are sufficiently similar due to the red color, and so we will call $P_{3}$ as red. However, it is also possible that our decision will be opposite. Then, since $P_{1}$ and $P_{3}$ would not be sufficiently similar due to the red color, $P_{3}$ would be called non-red. It seems necessary to emphasize that our decision does not need to be final - we can change it. There are many doubtful and difficult cases. In such cases it is possible that at one moment, two objects can be recognized by us as sufficiently similar due to the meaning of the name, and not recognized as such at some other moment. Moreover, objects observed by us just before the decision is taken influence the decision. Usually in such a situation, a pale red object in the presence of intensively red objects is recognized as non-red. Otherwise, white, black or green objects can provoke us to the opposite attitude. Finally, it is worth noticing that the lack of the sufficient similarity of some object to the given model examples of the correct use of the expression $A$ does not mean that the object is sufficiently similar to the model examples of the correct use of the expression not-A. Somebody can be for us neither bald nor not-bald. These states make us introduce (create) a new word(s) 
for adequate naming of all such dubious cases. The third leaf of paper from the example, can be called neither red, nor not-red but just "pale red", "almost red", "pink", "dimly red", "softly red”, "white-red”, etc.

It has been already mentioned that our opinion in such cases depends on our experience, knowledge, intuition, and probably on some other factors. But all the factors listed and not listed here depend on model examples we have in our memory or we had at one time. On the other hand, our opinions how to correctly use the name $A$ can be identified with our understanding of the meaning of $A$. Thus, our understanding of the meaning of $A$ depends on all our model cases of the correct use of the name $A$. In this sense, our understanding of the meaning of words is defined by model cases we have/had in our memory. Since other users of the language influence our choice of the model cases representing in our memory the meaning of words, meanings have social character. In this sense they are created by the community we live in. In two introductory stories (at the beginning of the paper) the role of community is played by the father.

Returning to the example with the three leaves of paper, due to the meaning of the name "leaf of paper" every two (from all three) objects are sufficiently similar, and due to the name "a leaf of paper overprinted by black letters on one side" objects $P_{1}$ and $P_{2}$ are sufficiently similar, but $P_{1}$ and $P_{3}$ as well as $P_{2}$ and $P_{3}$ are not sufficiently similar. The meaning of the name forces us to ignore all features, which are not related to the meaning in consideration. That is why, from the point of view of the meaning of some name $A$, two objects can be sufficiently similar although at first view they seem to be completely and undoubtedly dissimilar.

All these remarks are related to the process of knowing the meaning of these words, which already exist in the language, as well as of the extension of the language by new words naming objects from penumbra of some vague expressions. However, it is not settled how the natural language appears. That is why, there is assumed some "social" knowledge about the correct use of words, and a process of expansion of that knowledge. This "social" knowledge consists of two fundamental components: the sufficient similarity to the model examples and the always only partially given content of expressions. All above remarks dealt mainly with the first component. However, the second one is a fact, too. Unfortunately, we are not aware that accepted by us defining usually is partial only. We believe in the correctness and accuracy of definitions of many words, and all logical arguments (like sorites) which contradict these beliefs become a real shock. Defining meaning is partial because it is nothing more than the "verbal" counterpart 
of the "visual" concrete model example. For its concreteness, every visual model example can only partially represent all possible referents of the word. Similarly, every description of the meaning of the expression defined by words only partially explains the meaning. Aristotle's definition of a man as a rational animal is precise in a similar way as the father's definition of the shed: it is an object standing on the ground smaller than building, constructed from ordinary boards, not for living, just for garden's tools. Every partial defining refers to some concrete observable case and creates a picture of this case. Aristotle thought about people living around him, probably forgetting about people with heavy mental diseases. Similarly, the father looking at the concrete shed did not think about many other various sheds which do not coincide with his description. Usually, this partial defining of expressions leads to no serious problems. Moreover it is economical - communication is faster and simpler. Preciseness can always be increased depending on needs and context. Partial defining causes problems in one case only, when we try to treat the partial definition as the complete one. However, for no natural language expression $E$ there exists a complete definition, i.e. sentence correct definition of the form: for any $x, x$ is $E$ if and only if $x$ satisfies $b_{1}, \ldots, b_{k}$. Every finite characteristics of $E$ generates gaps in the scope of possible referents of $E$. This problem is closely related with the vagueness of natural language expressions, and is discussed in the next paragraph. This partial defining has some interesting result. Although we strongly believe in correctness, it means completeness of many definitions of words, in any problematic situation we know "very well" how to use the word totally ignoring the definition. For example, we believe that the man is a rational animal, but thinking about some deeply mentally subnormal man, who surely is unable to think, we ignore the definition and without doubts know very well, that he/she is a man.

This defining is also relative - it appeals to the meaning of already known words. Of course, already known words have meaning as precise as those partially defined. All non-mathematical expressions - the already known as well as the new once - have only partial understanding and are mutually relative. Unlike the shed, the building is enough big, it is possible to live in it, etc. The shed unlike the building is rather small and it is impossible to live in it. Such a mutually relative defining is more operative than the hierarchy of definitions with undefined primitive terms at the base. Always, understanding of new words appeals to the understanding of old ones. In this sense it is relative. But unlike the mathematical language, the natural one does not contain the so called primitive terms - terms which are introduced without definition. Although defining is relative in mathe- 
matics, not all terms are defined. In the natural language all terms can be defined, if we only wish. This defining is always relative and partial. That is why, unlike in mathematics, natural language expressions have approximate and rough understanding. It is not a problem, because it is temporal - understanding of words is still developed and improved. During their life the child will change old model examples onto better new model pictures of objects, and old partial definitions onto more precise but still partial new verbal characteristics. This process never ends and for two people neither proceeds in the same way nor results exactly in the same understanding of words.

\section{Vagueness - another face of the tolerance of expression}

In some moments in the above considerations there has appeared the problem of vagueness as a reason for inability of complete understanding/defining of all those natural language expressions which have no mathematical sense. Let us recall that the name $N$ is vague if and only if there are objects about which nobody can know if they are designates of $N$ or not. It means that for some object $O$ nobody can reasonably decide that it is a designate of $N$ or not a designate of $N$. More generally, an expression $E$ is vague, if and only if there are some cases that nobody can know (or reasonably decide) if $E$ or not-E can be correctly used as a name for these cases or not. All those doubtful cases create the so called penumbra of the expression also called the set of border-line cases (Russell, 1923, also Łukowski, 2011: 141-151). In fact, "penumbra" is a better name because this area of "vague" objects or cases is not a set in the mathematical sense as nobody can even know if some object or case belongs to penumbra or not. Penumbra has no sharp boundaries separating it from both extensions: positive and negative. Positive extension of $E$ consists of all those objects which are designates of $E$ or all those cases which can be correctly named by $E$. The negative extension of $E$ contains all those objects which are not designates of $E$ or all those cases which cannot be correctly named by $E$. Of course, penumbra of $E$ has no sharp borders if and only if at least one of the extensions of $E$ has no sharp borders. Moreover, an expression $E$ is vague if and only if an expression not- $E$ is vague.

There is a procedure, called sorites, which enables to verify whether the expression is vague. Sorites is an argumentation that uses two premises typical for 
mathematical induction. The first premise states that the expression $E$ applies to some object or case. Let us call of the property ascribed by $E$ to the object/ case a "feature". The second premise allows to transfer the feature from any object/case to the next one - all objects or cases are previously linearly ordered in such a way that the first object should be correctly named by $E$ (because it is $E$ ) and the last one should be correctly named be not- $E$ (because it is not-E). It is clear that, if both premises are satisfied the entire argumentation results in contradiction: the last object in the sequel is named by $E$, but it is not-E. Sorites consists of so small steps that passing from $E$ to not-E cannot be stopped at any other step because of the obvious correctness of every step. Thus, everything in the argumentation is obviously true with the exception of the conclusion which, as it leads to a contradiction, is obviously false. If the sorites applied to the expression $E$ leads to contradiction, it means that $E$ is vague. Russell, in his famous lecture given in 1923, pointed out the vagueness of many various natural language expressions (Russell, 1923). ${ }^{2}$ As it was showed by Black, all names of real, inanimate objects are vague (Black, 1949: 433). ${ }^{3}$ Chwistek showed that all names for living beings are vague (Chwistek, 1934: 10-11). ${ }^{4}$ Many years later, Sorensen showed that some abstract names also are vague (Sorensen, 1990). Shortly speaking almost all natural language names and predicates are vague, with the exception of: "motion", "rest" (i.e. not-motion), Parmenides's "being" and "not-being". It is impossible to pass from the case of movement to the case of rest through cases which are neither motion nor rest. Similarly, there is nothing which would be neither being nor not-being. The only surely sharp (i.e. not-vague) expressions are mathematical.

A tolerance is an essence of the vagueness. An expression cannot be vague not being tolerant. Tolerance of the expressions makes the second inductive premise of sorites true. In the sequel, every object or case and its successor as well as predecessor are sufficiently similar due the understanding of $E$. That is why, the reasoning cannot be stopped at any step. Such objects are sufficiently similar because a difference - due to the meaning of $E$ - between them is insignificant. If such difference between two objects or cases is insignificant, then only

2 Russell tried to prove that even logical terms are vague.

3 The argument showing, that the not-chair (a lump of the chair's leg) must be called a chair.

4 Chwistek asked his famous question: "Is human's mother a human?". In the light of the theory of evolution the answer on this question is not obvious.

5 Sorensen showed that "murder" is a vague name. Other abstract terms are also vague, e.g. "longing”, (Łukowski, 2008: 32-33).

\section{O) COGNITIVE SCIENCE}


two situations are reasonable: either both of them will be called $E$ by us or both of them will be called not-E. For insignificant difference it is excluded that one object/case will be called $E$ and the second not-E. Otherwise, if the difference is significant both objects/cases have to be called differently: if one is $E$, then, the second is not-E. It is clear that the inductive premise and the principle of the tolerance are strictly connected. Two names "sufficient similarity" and "insignificant difference" refer to the same. That is why the vagueness is a kind of display of the tolerance.

The second dialogue shows how the boy is taught by his father how to correctly use the vague name "red". Logical knowledge about vagueness warns us that such a use cannot be consequent and precise. Real cases are named by us by tolerant words whose understanding is established by model examples. However, all such examples can compose a sequent from the reasoning sorites. It is a real, unsolvable problem. If anybody from us will be called "human being", then his/ her mother also has to be called "human being" - it is obvious consequence of our meaning of the word "human being". However, all such selected ancestors compose the sequence imminently leading us to non-human beings. It is an effect of the confrontation of the real world with our limited natural language.

\section{Coexistence of generality and vagueness}

As it was shown, tolerance of natural language expressions occurs in two forms: generality and vagueness. In the case of vagueness, two objects are sufficiently similar due to some feature, if the feature is gradable in barely recognizable way - degrees of the feature should be small enough. Somebody with $n$ hairs on the head is sufficiently similar due to the understanding of the name "bald" to anybody with e.g. $n+36$ hairs. Then, if one of these persons will be called bald (not bald), then the second one should be called by the same name. This is a "vague" case of the tolerance. However, tolerance does not need to be connected with only gradable features. Then, the tolerance has a face of generality of expressions. Let us assume that $A$ is a father of a girl, while $B$ is a father of a boy. Due to the understanding of the name "father" $A$ and $B$ are sufficiently similar. The scope of this name can be divided into sub-scopes: "father of one girl", "father of two girls"..., "father of a boy", "father of two boys" ..., "father of a girl and a boy", "father of two girls and one boy"... It is easy to notice, that the possible order 
of the set of all these sub-scopes can be partial, but not linear. That is why, it is difficult to consider the feature "to be a father" as gradable. However, regardless of the possible order, all sub-scopes consist of cases which are sufficiently similar: due to the meaning of the word "father" the father of two girls and one boy is sufficiently similar to the father of three boys - a difference due to the meaning (i.e. correct understanding) of the name "father" is insignificant. Even, if all these subscopes would have sharp boundaries there would be still a tolerance - a "general" case of the tolerance. However, from the practice of medicine we know that all these sub-scopes have not sharp but vague borders - a difference between two fathers does not need to be gradable. It means that the name "to be a father" of the feature is a case of tolerance of the mix type simultaneously coming from vagueness and from generality of the word "father".

Intermingling both kinds of tolerance can be also easily exemplified by the word "red". Two objects a red t-shirt and a red car are sufficiently similar due to the understanding of the word "red" - it does not matter that one is a t-shirt and another a car. Both objects belong to the scope of the general name "red (object)". This scope can be divided into many subsets (sub-scopes) represented by names: "red car", "red t-shirt", "red dress", "red umbrella"... However, it does not matter from which subsets two objects come, both are sufficiently similar due to the word "red". Apart from this "general" case of the tolerance there is another, "general" case. Indeed, the scope of the word "red" can be divided in another, "vague" way into subsets represented by names: "bright red", "dark red", "deep dark red”, "red with yellow tone", "red with slight yellow tone"... Surely, these sub-scopes are not distinguished by any sharp borders. It is not a difficult exercise to imagine the situation joining both kinds of tolerance: the light-red car and the washy-red t-shirt.

Fortunately, usually we meet such objects and cases which can be clearly distinguished from the point of view the understanding of words. In the first dialogue, the child is looking at the shed which differs gradually from other objects standing around. It is not difficult to imagine a long sequence of objects changing in barely recognizable way from the concrete shed to the concrete building. Then, it would be a problem to name all such objects. Fortunately, in our life we have simpler cases that explicitly differ from each other, like the child, for whom the building clearly differs from the shed. Because of this fact, the father can partially (and relatively) define both names "building" and "shed" - such defining is sufficient.

While observing a new object we need to compare it due to the meaning of the given name with some model example in our memory. Depending on the kind of similarity we have to do with the "vague" or "general" tolerance. Although the

\section{O) COGNITIVE SCIENCE}


name can be simultaneously vague and general (of course, each from another point of view) one of the tolerances should be ignored. We treat the name either as tolerant in the sense of vagueness or as tolerant in the sense of generality. Our choice depends on the character of the feature due to which we recognize the similarity.

\section{Conclusion}

Procedures expanding linguistic competence can be divided into two kinds. The first teaches us how to correctly use general expressions, the second how to correctly use vague expression. Both procedures differ in some essential way, because there is an essential difference between generality and vagueness. However, both procedures have some common features. Firstly, both use linguistic competence of the community. In this sense, our understanding and use of words has a social character. No already existing word can be successfully used in violation of the existing use of this word in the community. Secondly, both procedures have an ostensive character - it does not matter, if it is a red color or a building and a shed. Every procedure appeals to the model examples we have in our memory. All these examples play a role of useful, concrete types of these cases in which we can undoubtedly and correctly use the word. Thirdly, both procedures only lead to a partial characterization of the correct use of words. One procedure prefers visual characterization, while the other verbal one. Both characterizations are not adequate to the full range of the correct use of the words and so they are not correct. Because of this fact, they are still improved and frequently changed - still with the help of members of the community. So, partiality implies temporality.

Procedure extending linguistic competence by vague expressions will be here called the "procedure for vagueness teaching". Its schema has been already discussed above. Recapitulating, if someone would like to take a correct decision to use or not to use the word "red" in a given case, he/she would compare the color of the new object to the model examples existing in the memory. If the similarity seems to be sufficient, the name "red" can be used. In other situations, it is necessary to use some extended method. Since the color of the new object can be neither red nor not-red, it means that it is a tone of some other color, e.g. brown. Then, it is useful to recognize to which from these two colors (red and brown) represented by some model examples the new color is more similar. Then, we can say that the new color is brown-red but it is more red that brown - it is more similar to the model of the red color than to the model of the brown color. 


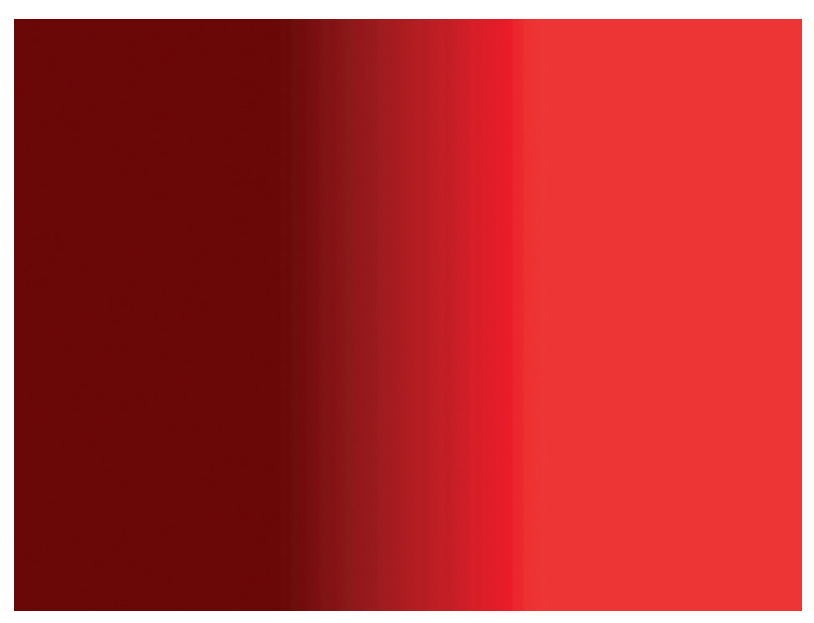

Fig. 1

This procedure has some concrete exemplification in the form of the so called conceptual spaces invented by Gärdenfors and based on Voronoi tessellation (also named Voronoi partition) (Gärdenfors, 2000, 2014, see also Voronoi, 1908). Thanks to Gärdenfors's modification it is an interesting scheme to consider the penumbra of vague names. Initially, the distinction between two colors is sharp, exactly due to the Voronoi partition, being an axial symmetric line between two points representing the model examples.

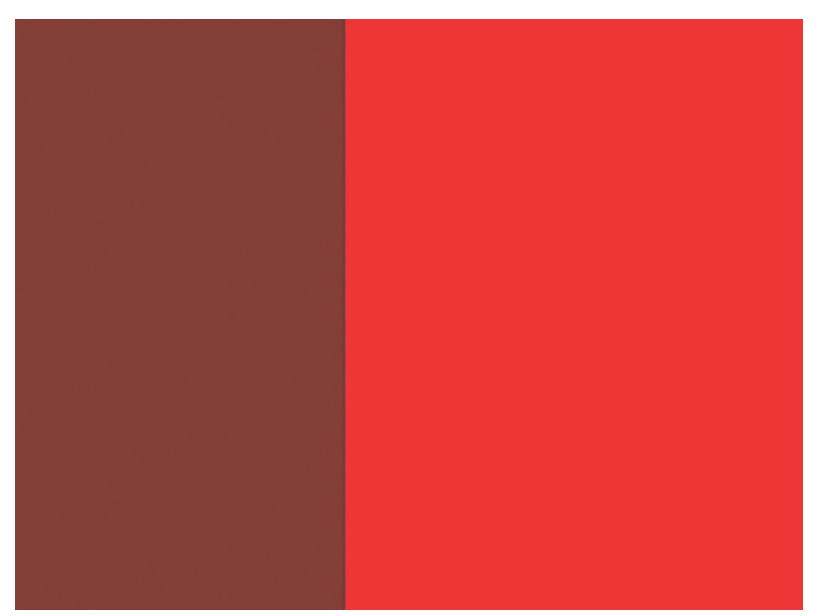

Fig. 2.1 


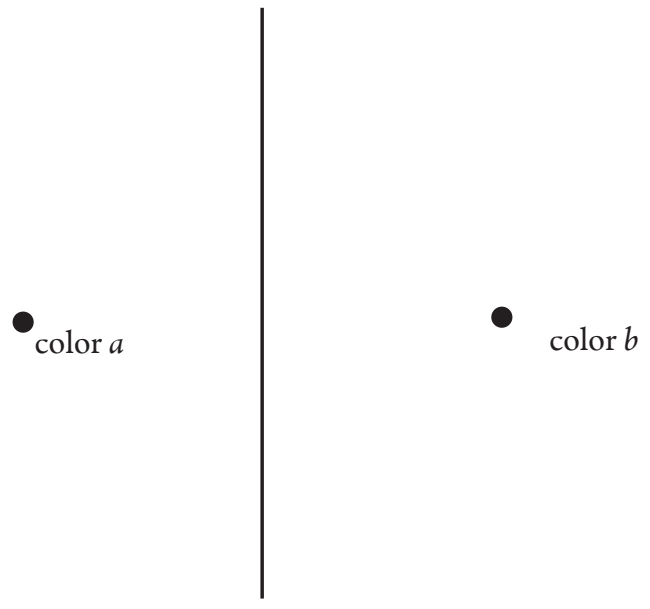

Fig. $2.2^{6}$

However, it suffices to use more than one model example for at least one color (for example, only red, and not brown) and sharp border is replaced by the bundle of several sharp borders, which taken together give a vague border, i.e. penumbra. ${ }^{7}$

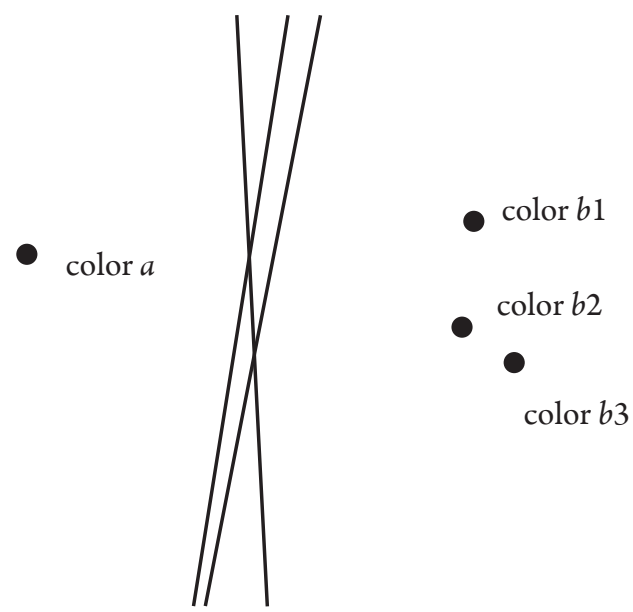

Fig. 3

${ }^{6}$ Fig. 2.2 is a schema of the previous fig. 2.1.

7 This graphic model precisely coincides with the class of precisifications - every sharp line from the graph is just a one precisification function (see: van Frassen, 1966, 1968; Fine, 1975). 
Unfortunately, it is impossible to "return" to one colored picture related to the fig. 3. Instead of one figure it should be here, three pictures, respectively to pairs: $(a, b 1),(a, b 2),(a, b 3)$. All of them taken together should create a three-layer picture, where every layer presents its own sharp border between $a$ and $b i$, for $i=1,2,3$.

Summarizing, let us reconstruct the scheme of the procedure on the example of the second story. The agent (the child) gives information from the community (from the father) that the color of the father's jacket is brown. In the agent's memory there appears a record of the model example of the brown color. In a similar way, it appears in his/her memory another model example of the brown color (leaf). Both examples are different, so in the memory color brown is represented by more than one model example. Thus, the brown color has not one rigid, precise understanding. Since all these examples are given by the community, the agent's understanding of the correct use of the word "brown" is social. After some time, the agent needs to recognize the color of that car. The color of the new object is not represented in the agent's memory, but it is similar to the brown. With the help of the father, the child tries to decide, if the color is more similar to brown or to red - at this stage of the procedure the help of the father is not necessary. If the new color will be more similar to red, the agent will call it red, if it will be more similar to brown, it will be named red. But, there is another third possibility. The agent can decide that the new color is brown-red. However, the decision is not easy, because neither red nor brown are represented by single model examples. It means that there is a vague area of all these cases which are neither red nor brown (see fig. 3 ). After some time, the agent is able to introduce to his memory new model examples of the brown color - all these colors which are (in the picture) between model examples of the brown.

As it has been already mentioned, colors are not the only vague terms. There are plenty of vague expressions in the natural language. However, the procedure of recognizing of the correct use of the vague name/predicate seems to be standard and similar to the just presented. The problem of the vagueness should be in the procedure appropriately separated from other problems, e.g. generality. If we need to recognize which of two objects, a house and a chair, is wooden, we ignore all differences coming from the fact that one is a house and the other a chair. In both cases it can be difficult to decide, if some of them is wooden or not.

Procedure extending a linguistic competence by general expressions will be here called the "procedure for generality teaching". Similarly to the previous 
procedure, this one also employs model examples, mainly at the first moment, but not only. Thus, firstly, the agent calls the object by the name "building" because of some features common to the new object and to the picture of the building. It is a mistake, which is immediately revised by the father - a member of the community. In the next step, the father calls the new object by the name "shed". In the case of the vague term it could be the end of teaching. Here, however, the father formulates a list of features which make an average building distinct from an average shed. ${ }^{8}$ This partial, relative and verbal defining of the shed will constitute the correct use of the name "shed" as well as that of "building" in the future. Of course, probably this partial characterization will be improved by a better list, which also will be a partial only defining. Such a partial defining is a logical necessity although it is the reason of mistakes occasionally made by people using it. Let us assume that $O$ is partially defined as a name of an object being $a_{1}, a_{2}, a_{3}, a_{4}$, and not being $b_{1}$, $b_{2}, b_{3}$ (i.e. being $\neg b_{1}, \neg b_{2}, \neg b_{3}$ ). Since it is partial defining of $O$ the set $\left\{a_{1}, a_{2}, a_{3}, a_{4}\right.$, $\left.\neg b_{1}, \neg b_{2}, \neg b_{3}\right\}$ does not suffice as a complete characterization. It means that in the future we might meet an object which should be named $O$, and its characterization is a little bit different: $a_{1}, a_{2}, a_{5}, a_{4}, \neg b_{1}, \neg b_{2}, \neg b_{3}, \neg b_{4}$, i.e. instead of $a_{3}$ there are added $a_{5}$ and $\neg b_{4}$. Now, we have two alternative definitions, and both are partial - improvement of the partial definition leads to the next partial definition, because the complete definition of $O$ does not exist. Obviously, all definitions are temporal - in the future every definition can be made more precise or even rejected.

Two, considered here, procedures expanding a linguistic competence have some important similarities although the first one deals with vagueness, while the second with generality. Inhelder and Piaget noticed that during our childhood original accidental similarities are consequently replaced by more conceptually advanced essential similarities (see: Keil, 1989: 9, also Piaget, Inhelder, 1974: 37). Every procedure:

1) is a social phenomenon thanks to Vygotsky's zone of proximal development (Keil, 1989: 5-7);

2) is based on model examples - "verbal" or "visual" - permanently replaced by better once;

3) leads to a merely partial defining, which can be "verbal" or "visual";

4) is dynamic and temporal.

8 To speaking the truth, also in the previous case the father tries to explain the difference formulating the feature which is an essence of the difference: that color is not brown because it has a red savor. 
From this perspective, a meaning of the natural language expression is identified with the correct, i.e. accepted by the community, use of this expression. It means that the meaning of words is social, partial, and temporal, where partial means imprecise and misleading, while temporal means dynamic and changing.

\section{References}

Black, M. (1949). Language and Philosophy. Studies in Method, Ithaca-New York: Cornell University Press.

Chwistek, L. (1934). Granice zdrowego rozsądku. In: L. Chwistek, Pisma filozoficzne i logiczne, vol. 2, Warszawa: PWN, 1963.

Fine, K. (1975). Vagueness, truth and logic. Synthese 30: 265-300.

Gärdenfors, P. (2000). Conceptual Spaces. Cambridge MA: MIT Press.

Gärdenfors, P. (2014). Geometry of Meaning. Semantics Based on Conceptual Spaces. Cambridge MA: MIT Press.

Keil, F.C. (1989). Concepts, Kinds and Cognitive Development. Cambridge: MIT Press.

Łukowski, P. (2008). Paradoksy nieostrości i zmiany. Toruń: Wydawnictwo Rolewski.

Łukowski, P. (2011). Paradoxes. London-New York: Springer.

Łukowski, P. (2012). Logika praktyczna z elementami wiedzy o manipulacji. Warszawa: Wolters Kluwer.

Maruszewski, T. (2001). Psychologia poznania. Gdańsk: Gdańskie Wydawnictwo Psychologiczne.

Piaget, J., Inhelder B. (1974). The child's construction of quantities. London: Routledge and Kegan Paul.

Russell, B. (1923). Vagueness. The Australasian Journal of Psychology and Philosophy 1 (4): 84-92.

Sorensen, R. (1990). Vagueness implies cognitivism. The American Philosophical Quarterly 27 (1): 1-14.

van Frassen, B.C. (1966). Singular terms, truth value gaps and free logic. Journal of Philosophy 63: 481-495.

van Frassen, B.C. (1968). Presupposition, implication and self-reference. Journal of Philosophy 65: 136-152.

Voronoi, G. (1908). Nouvelles applications des paramètres continus à la théorie des formes quadratiques. Journal für die Reine und Angewandte Mathematik 133: 97-178. 